\section{Reflujo vesicoureteral sintomático posterior al injerto renal en adultos: tratamiento endourológico y reacción con agentes bloqueantes}

Gutiérrez-Jiménez AA, ${ }^{1,2}$ Jiménez-López LA, ${ }^{1}$ Ricardez-Espinosa AA, ${ }^{1,2}$ GeorgeMicceli E, ${ }^{1}$ Aguilar-Sandoval EG, ${ }^{1}$ Bravo-Duarte $\mathrm{GA},{ }^{2}$ y colaboradores.

\begin{abstract}
Resumen
El reflujo vesicoureteral después del trasplante renal es una complicación común, que puede favorecer la aparición de infección de vías urinarias recurrente y cuadros de pielonefritis aguda en el injerto, lo que incrementa el riesgo de su pérdida. Por consenso general, los pacientes con reflujo vesicoureteral sintomático deben recibir tratamiento. El tratamiento endourológico ha demostrado curar el reflujo y disminuir el índice de infección de vías urinarias y pielonefritis con pocas complicaciones. En las últimas décadas se han prescrito diferentes fármacos e implementado diversas técnicas para el tratamiento del reflujo vesicoureteral sintomático posterior al trasplante renal. Este artículo revisa las diferentes técnicas utilizadas y analiza la eficacia de los distintos fármacos prescritos para el reflujo vesicoureteral sintomático posterior al trasplante renal en adultos.
\end{abstract}

PALABRAS CLAVE: agentes bloqueantes, agentes expansores, reflujo vesicoureteral sintomático, trasplante renal, injerto renal.

Rev Mex Urol. 2017 Sep-Oct;77(5):419-424.

\section{Symptomatic vesicoureteral reflux after kidney graft in adults: endourological management with blocking agents and their results}

Gutiérrez-Jiménez AA, ${ }^{1,2}$ Jiménez-López LA, ${ }^{1}$ Ricardez-Espinosa AA, ${ }^{1,2}$ GeorgeMicceli E, ${ }^{1}$ Aguilar-Sandoval EG, ${ }^{1}$ Bravo-Duarte $\mathrm{GA},{ }^{2}$ y colaboradores.

\section{Abstract}

Vesicoureteral reflux after kidney transplant is a common complication and can favor recurrent urinary tract infection and acute pyelonephritis in the graft, increasing the risk for graft loss. The general consensus is that symptomatic vesicoureteral reflux should be resolved. Endourologic management is recommended because it has shown reflux

\author{
${ }^{1}$ Departamento de Urología, Unidad Médica de Alta \\ Especialidad, Hospital de Especialidades 14, Centro \\ Médico Nacional Adolfo Ruiz Cortines, Instituto Mexi- \\ cano del Seguro Social, Veracruz, México. \\ ${ }^{2}$ Facultad de Medicina Miguel Alemán Valdés, Uni- \\ versidad Veracruzana. \\ Recibido: marzo 2017 \\ Aceptado: agosto 2017 \\ Correspondencia \\ Dr. Ángel Armando Gutiérrez Jiménez. \\ angelgj83@gmail.com
}

Este artículo debe citarse como

Gutiérrez-Jiménez AA, Jiménez-López LA, RicardezEspinosa AA, George-Micceli E, Aguilar-Sandoval EG, Bravo-Duarte GA, y col. Reflujo vesicoureteral sintomático posterior al injerto renal en adultos: tratamiento endourológico y reacción con agentes bloqueantes. Rev Mex Urol. 2017 sepoct;77(5):419-424.

DOI: https://doi.org/10.24245/revmexurol.v77i5.1371 
resolution, a reduced rate of urinary tract infections, and pyelonephritis with few complications. Over past decades, a variety of agents and procedures have been used for managing symptomatic vesicoureteral reflux after kidney transplantation. The present article reviews the characteristics of the different techniques utilized and analyzes the efficacy of the different products employed for the endoscopic resolution of symptomatic vesicoureteral reflux after kidney transplantation in adults.

KEYWORDS: Blocking agents; Expanders; Symptomatic vesicoureteral reflux; Kidney transplantation; Kidney graft
1 Departamento de Urología, Unidad Médica de Alta Especialidad, Hospital de Especialidades 14, Centro Médico Nacional Adolfo Ruiz Cortines, Instituto Mexicano del Seguro Social, Veracruz, México.

2 Facultad de Medicina Miguel Alemán Valdés, Universidad Veracruzana.

Correspondence Dr. Ángel Armando Gutiérrez Jiménez. angelgj83@gmail.com

\section{ANTECEDENTES}

El reflujo vesicoureteral posterior al trasplante renal es una complicación común, pues se estima que afecta de 40.7 a $86.4 \%$ de los pacientes. ${ }^{1-3}$ La elevada tasa de reflujo vesicoureteral quizá sea provocada por la técnica quirúrgica, debido a que en algunos casos se prefiere realizar un implante amplio, con la finalidad de prevenir la formación de estenosis. ${ }^{4}$ Cuando el reflujo vesicoureteral se asocia con cuadros de pielonefritis aguda al injerto se considera sintomático, incluso en 1.1 a $2 \%$ de los pacientes intervenidos de trasplante renal. ${ }^{5-8}$ El reflujo vesicoureteral posterior al injerto se diagnostica mediante cistograma miccional, con grado de afectación variable, según la clasificación del Consenso Internacional. ${ }^{9}$

En el año 2000, Masahiko y sus colaboradores realizaron un estudio comparativo que incluyó a 1187 pacientes posoperados de trasplante renal, en el que analizaron las complicaciones mediante la técnica transvesical Politano-Leadbetter versus extravesical Lich-Gregoir. El estudio reportó una incidencia de reflujo vesicoureteral sintomático de 1.5 y $0.8 \%$, respectivamente, sin encontrar diferencias estadísticamente significativas. Dos años después, Secin y su grupo no mostraron evidencia de reflujo sintomático en los pacientes a quienes se practicó la técnica de reimplante ureteral de Taguchi. La misma observación fue descrita por Nie y su equipo de trabajo, al utilizar ureteroneostomía..$^{5,10-12}$ Entre los factores de riesgo para reflujo vesicoureteral se encuentran: capacidad vesical disminuida previa al trasplante y falta de experiencia del cirujano. ${ }^{1,13}$ Los pacientes con infección de vías urinarias después del trasplante renal tienen mayor riesgo de pérdida del injerto y mortalidad. ${ }^{14}$

Dupont y su grupo encontraron que los pacientes con infección de vías urinarias y reflujo vesicoureteral posterior a trasplante renal tuvieron mayor incidencia cicatrices renales que quienes no tuvieron reflujo. ${ }^{15}$ Pellé y sus colaboradores reportaron que los episodios de pielonefritis aguda se correlacionan con la frecuencia en que aparecen las infecciones de vías urinarias y los episodios de rechazo del injerto. ${ }^{16}$

Por consenso general, los pacientes con reflujo vesicoureteral sintomático deben someterse a tratamiento. La corrección quirúrgica sigue 
siendo la técnica de referencia; sin embargo, debido a la baja morbilidad de la endoscopia, se recomienda como primera opción de tratamiento y se prefiere la corrección quirúrgica abierta solo en los casos en quienes ha fallado el tratamiento endoscópico. ${ }^{4,17}$

\section{Tratamiento endourológico del reflujo vesicoureteral posterior al injerto renal}

\section{Técnicas para la inyección de agentes bloqueantes}

Entre las técnicas actuales para la corrección del reflujo vesicoureteral sintomático se encuentran: STING (por su siglas en inglés: subureteric transurethral injection), descrita en 1981 por Matouschek, quien sugiere ingresar en la mucosa de la vejiga, entre 2-3 mm distal a la unión del uréter (UUV), y realizar un avance de la aguja en el plano submucoso, a una distancia de 4-5 $\mathrm{mm}$, aplicando el agente bloqueante hasta la coaptación del orificio ureteral. ${ }^{18,19}$ La técnica STING modificada, popularizada por Kirsch y su grupo, se efectúa con hidrodistensión (dirigiendo la corriente de flujo de la irrigación al uréter), al insertar la aguja a nivel subureteral, aproximadamente $4 \mathrm{~mm}$ dentro de la submucosa; la primera aplicación se realiza en posición de las 6, según la manecillas del reloj, del orificio ureteral y con ello debe lograrse que el piso del túnel ureteral alcance su techo. Posteriormente se aplican inyecciones adicionales, circunferencialmente, hasta obtener la coaptación completa del meato. ${ }^{20}$ Por último, la técnica de HIT (hydrodistention-implantation), descrita en 2007 por McMann y sus colaboradores, ${ }^{21}$ incluye la aplicación de dos inyecciones submucosas intraureterales, una proximal y otra distal (doble HIT), hasta la coaptación total del túnel ureteral. Estas técnicas fueron descritas, inicialmente, para el tratamiento del reflujo vesicoureteral primario en niños y posteriormente para el reflujo vesicoureteral después del injerto renal. Yucel y su equipo compararon las técnicas de inyección in- traureteral versus STING modificada para evaluar el tratamiento del reflujo sintomático después del trasplante renal. ${ }^{22}$ Estos autores aplicaron un copolímero de dextranómero en ácido hialurónico $\left(\right.$ Deflux ${ }^{\circledR}$ ) y encontraron una tasa de éxito de $52.9 \%$ para la técnica intraureteral y de $55.5 \%$ para STING modificada; sin embargo, no reportaron diferencias estadísticamente significativas.

\section{Agentes bloqueantes}

Los estudios relacionados con la administración de agentes bloqueantes para el tratamiento del reflujo vesicoureteral sintomático posterior al injerto renal son escasos. Este tipo de fármacos incluye agentes sintéticos, por ejemplo: copolímero de dextranómero en ácido hialurónico $\left(\right.$ Deflux $\left.^{\circledR}\right),{ }^{23-25}$ politetrafluoroetileno (Polytef $\left.{ }^{\circledR}\right),{ }^{26,27}$ carbón pirolítico (Durasphere $\left.{ }^{\circledR}\right)^{28}$ y polidimetilsiloxano (Macroplastique ${ }^{\circledR}$ ); ${ }^{29,30}$ entre los agentes biológicos, el más común es el colágeno purificado bovino. ${ }^{31}$

\section{Resultados o reacción de los agentes} bloqueantes

Las definiciones de éxito en los diferentes estudios son variables; mientras algunos definen como éxito la ausencia de pielonefritis aguda por injerto entre 1-2 años posimplante (éxito clínico), otros señalan ausencia de reflujo vesicoureteral en el cistograma de control (éxito radiológico). ${ }^{29,30} \mathrm{La}$ mayor parte de los estudios reporta adecuada reacción con el tratamiento endoscópico, con una sola aplicación del agente bloqueante; sin embargo, cuando no se obtienen resultados satisfactorios (éxito), puede indicarse más de una aplicación durante el seguimiento, para que de esta forma se obtenga una tasa de éxito global. El Cuadro 1 describe los principales estudios publicados en la actualidad y las tasas de éxito global de los diferentes medicamentos. Otro punto importante en cuanto a la interpretación de los resultados es el tiempo de seguimiento, 
Cuadro 1. Eficacia del tratamiento endoscópico con agentes bloqueantes

\begin{tabular}{|c|c|c|c|c|c|c|c|}
\hline $\begin{array}{l}\text { Autor, año de } \\
\text { publicación }\end{array}$ & $\begin{array}{c}\text { Agente } \\
\text { bloqueante }\end{array}$ & $\begin{array}{l}\text { Población } \\
\text { (n) }\end{array}$ & $\begin{array}{c}\text { Media de } \\
\text { edad } \\
\text { (años) }\end{array}$ & $\begin{array}{c}\text { Técnica utilizada para } \\
\text { el implante }\end{array}$ & $\begin{array}{l}\text { Periodo de } \\
\text { seguimiento }\end{array}$ & Complicación & $\begin{array}{c}\text { Tasa } \\
\text { global } \\
\text { de éxito }\end{array}$ \\
\hline $\begin{array}{l}\text { Song y col. } \\
(2011)^{29}\end{array}$ & Macroplastique & 19 & 50.6 & $\begin{array}{c}\text { Ureteroneocistostomía } \\
\text { extravesical }\end{array}$ & $\mathrm{N} / \mathrm{D}$ & Ninguna & $63.2 \%$ \\
\hline $\begin{array}{l}\text { Nison y col. } \\
(2016)^{32}\end{array}$ & Macroplastique & 48 & 50 & N/D & 25 meses & Ninguna & $70.6 \%$ \\
\hline $\begin{array}{l}\text { Akiki y col. } \\
(2015)^{30}\end{array}$ & $\begin{array}{l}\text { Macroplastique } \\
\text { Deflux }\end{array}$ & 58 & 52 & Lich-Gregoir & 30.5 meses & Ninguna & $56.1 \%$ \\
\hline $\begin{array}{l}\text { Pichler y col. } \\
(2010)^{23}\end{array}$ & Deflux & 19 & 48 & $\begin{array}{c}\text { Politano-Leadbetter y } \\
\text { Lich-Gregoir }\end{array}$ & 6.5 meses & $\begin{array}{c}\text { Obstrucción } \\
\text { ureteral ( } 2 \\
\text { casos) }\end{array}$ & $78 \%$ \\
\hline $\begin{array}{l}\text { Yucel y col. } \\
(2010)^{22}\end{array}$ & Deflux & 26 & 32.2 & Lich-Gregoir & 3 meses & Ninguna & $53.8 \%$ \\
\hline $\begin{array}{l}\text { Romero y col. } \\
(2010)^{24}\end{array}$ & Deflux & 7 & 62 & $\mathrm{~N} / \mathrm{D}$ & 5 meses & $\begin{array}{l}\text { Hematuria } \\
\text { ( } 2 \text { casos })\end{array}$ & $85 \%$ \\
\hline $\begin{array}{l}\text { Antonopoulos } \\
\text { y col. }(2011)^{28}\end{array}$ & Durasphere & 8 & 38.8 & Lich-Gregoir & 23.2 meses & Ninguna & $75 \%$ \\
\hline $\begin{array}{l}\text { Cloix y col. } \\
(1993)^{26}\end{array}$ & Polytef & 21 & 37 & $\begin{array}{l}\text { Campos Freire y } \\
\text { Politano-Leadbetter }\end{array}$ & 10.7 meses & Ninguna & $30 \%$ \\
\hline $\begin{array}{l}\text { Latchamsetty y } \\
\text { col. }(2003)^{31}\end{array}$ & Colágeno & 7 & 47.3 & N/D & 2 meses & Ninguna & $0 \%$ \\
\hline
\end{tabular}

N/D: no descrito.

después de la aplicación del agente bloqueante, que según el estudio de Yucel y su grupo puede ser tan corto como 3 meses ${ }^{22}$ o el de Akiki y sus colaboradores que reportan más de 30 meses..$^{30}$

El agente bloqueante sintético más prescrito para el tratamiento del reflujo vesicoureteral posterior al injerto renal es el copolímero de dextranómero en ácido hialurónico. La mayor parte de los estudios reporta una muestra reducida de pacientes, con una tasa de éxito que varía de 53.8 a $85 \%{ }^{22,24}$ El estudio más representativo es el de Yucel y su grupo, ${ }^{22}$ efectuado en 26 casos con media de edad de 32.2 años, quienes obtuvieron una tasa de éxito global de 53.8 a 3 años de experiencia-seguimiento en la corrección endoscópica del reflujo vesicoureteral sintomático posterior al trasplante renal.

El segundo agente con mayor indicación es polidimetilsiloxano. En el estudio de Song y su grupo, ${ }^{29}$ quienes utilizaron la técnica de STING, aplicaron polidimetilsiloxano en 19 pacientes y obtuvieron una tasa de éxito global de $63 \%$; además, observaron que esa misma tasa fue diferente según los distintos grados de reflujo vesicoureteral: $50 \%$ en el grado I, $33 \%$ en el grado II, $75 \%$ en el grado III y $67 \%$ en el grado IV. Estos hallazgos demostraron la eficacia del polidimetilsiloxano en pacientes con reflujo vesicoureteral de alto grado. Por su parte, Nison y sus colaboradores $^{32}$ aplicaron polidimetilsiloxane en 48 pacientes (51 injertos) y reportaron una tasa de éxito global de $70.6 \%$. El carbón pirolítico fue evaluado por Antolopoulos y sus coautores, quienes reportaron en su serie de 8 pacientes, con media de edad de 38.8 años, una tasa de éxito global de $75 \%$ con una sola aplicación. ${ }^{28} \mathrm{El}$ politetrafluoroetileno para el tratamiento del reflujo vesicoureteral sintomático posterior al injerto fue descrito por Cloix y sus colaboradores en 1993, quienes evaluaron 19 pacientes (21 injertos) y 
obtuvieron una tasa de éxito global de $30 \%$, con seguimiento a 5 años. ${ }^{26}$ Por último, el colágeno purificado bovino (agente bloqueante biológico), evaluado por Latchamsetty y su equipo de trabajo, quienes lo aplicaron en 7 pacientes, no lograron obtener resultados satisfactorios en el tratamiento del reflujo vesicoureteral en ningún caso $^{31}$ (Cuadro 1).

\section{Complicaciones asociadas con la aplicación de agentes bloqueantes}

Aunque históricamente se ha descrito la migración extralesional del agente bloqueante como principal complicación, ${ }^{33}$ ninguno de los autores la señala al respecto. Las complicaciones más frecuentemente descritas en sus estudios fueron: obstrucción ureteral provocada por el copolímero de dextranómero en ácido hialurónico, principalmente por la administración de volúmenes altos (mayor de $4 \mathrm{~mL}$ ), y hematuria después de efectuar el procedimiento. $^{23,24}$

\section{CONCLUSIONES}

La aplicación endoscópica de agentes bloqueantes sintéticos para el tratamiento del reflujo vesicoureteral sintomático posterior al injerto renal es efectiva en la mayoría de los pacientes, particularmente con copolímero de dextranómero en ácido hialurónico $\left(\right.$ Deflux $\left.^{\circledR}\right)$, carbón pirolítico (Durasphere ${ }^{\circledR}$ ) y polidimetilsiloxano (Macroplastique $^{\circledR}$ ). Estos agentes reportan tasas de éxito similares y baja morbilidad; sin embargo, se requieren estudios adicionales, con mayor número de pacientes y seguimiento a largo plazo para determinar su eficacia.

\section{REFERENCIAS}

1. Margreiter M, Györi GP, Böhmig GA, Trubel S, Mühlbacher $F$, Steininger R. Value of routine voiding cystourethrography after renal transplantation. Am J Transplant 2013;13(1):130-135.
2. Jung GO, Chun JM, Park JB, Choi GS, Kwon CH, Joh JW, et al. Clinical significance of posttransplantation vesicoureteral reflux during short-term period after kidney transplantation. Transplant Proc 2008;40(7):2339-2341.

3. Mastrosimone S, Pignata G, Maresca MC, Calconi G, Rabassini A, Butini R, et al. Clinical significance of vesicoureteral reflux after kidney transplantation. Clin Nephrol 1993;40:38-45.

4. Duty BD, Conlin MJ, Fuchs EF, Barry JM. The current role of endourologic management of renal transplantation complications. Adv Urol 2013;2013:246520.

5. Nie ZL, Zhang KQ, Li QS, Jin FS, Zhu FQ, Huo WQ. Urological complications in 1,223 kidney transplantations. Urol Int 2009;83(3):337-341.

6. Al-Shaer MB, Al-Midani A. The management of urological complications in renal transplant patients. Saudi J Kidney Dis Transplant 2005;16(2):176-180.

7. Dinckan A, Tekin A, Turkyilmaz S, Kocak H, Gurkan A, Erdogan $\mathrm{O}$, et al. Early and late urological complications corrected surgically following renal transplantation. Transpl Int 2007;20(8):702-707.

8. Martínez-Mier G, Jiménez-López LA, Valencia-Mercado D, George-Micelli E, Salas-Díaz FA, González-Medina MF. Urological complications following kidney transplantation using Lich-Gregoir technique: A 4-year experience in Mexico. Cir Cir 2009;77(2):111-114.

9. Lebowitz RL, Olbing H, Parkkulainen KV, Smellie JM, Tamminen-Möbius TE. International system of radiographic grading of vesicoureteric reflux. International Reflux Study in Children. Pediatr Radiol 1985;15(2):105-109.

10. Masahiko H, Kazunari T, Tokumoto T, Ishikawa N, Yagisawa T, Toma H. Comparative study of urosurgical complications in renal transplantation: intravesical versus extravesical ureterocystoneostomy. Transplant Proc 2000;32(7):1844-1846.

11. Nie Z, Zhang K, Huo W, Li Q, Zhu F, Jin F. Comparison of urological complications with primary ureteroureterostomy versus conventional ureteroneocystostomy. Clin Transplant 2010;24(5):615-619.

12. Secin FP, Rovegno AR, Marrugat RE, Virasoro R, Lautersztein GA, Fernandez H. Comparing Taguchi and Lich-Gregoir ureterovesical reimplantation techniques for kidney transplants. J Urol 2002;168(3):926-930.

13. Farr A, Györi G, Mühlbacher F, Husslein P, Böhmig GA, Margreiter M. Gender has no influence on VUR rates after renal transplantation. Transpl Int 2014;27(11):1152-1158.

14. Abbott KC, Swanson SJ, Richter ER, Bohen EM, Agodoa LY, Peters TG, et al. Late urinary tract infection after renal transplantation in the United States. Am J Kidney Dis 2004;44(2):353-362.

15. Dupont PJ, Psimenou E, Lord R, Buscombe JR, Hilson AJ, Sweny P. Late recurrent urinary tract infections may produce renal allograft scarring even in the absence of symptoms or vesicoureteric reflux. Transplantation 2007;84(3):351-355. 
16. Pellé G, Vimont S, Levy PP, Hertig A, Ouali N, Chassin C, Arlet $\mathrm{G}$, et al. Acute pyelonephritis represents a risk factor impairing long-term kidney graft function. Am J Transplant 2007;7(4):899-907.

17. Karam G, Kälble T, Alcaraz A, Aki FT, Budde K, Humke U, et al.Guidelines on renal transplantation; European Association of Urology; 2014. [En línea]. Dirección URL: <http:// uroweb.org/wp-content/uploads/27-Renal-Transplant LRV2-May-13th-2014.pdf>. [Consulta: 21 de mazo 2016].

18. Matouschek E. Treatment of vesicorenal reflux by transurethral teflón-injection. Urologe A 1981;20(5):263-264.

19. Hayn MH, Smaldone MC, Ost MC, Docimo SG. Minimally invasive treatment of vesicoureteral reflux. Urol Clin North Am 2008;35(3):477-488.

20. Kirsch AJ, Perez-Brayfield M, Smith EA, Scherz HC. The modified sting procedure to correct vesicoureteral reflux: improved results with submucosal implantation within the intramural ureter. J Urol 2004;171:c2413-2416.

21. McMann LP, Scherz HC, Kirsch AJ. Long-term preservation of dextranomer/hyaluronic acid copolymer implants after endoscopic treatment of vesicoureteral reflux in children: a sonographic volumetric analysis. J Urol 2007;177(1):316320.

22. Yucel S, Akin Y, Celik O, Erdogru T, Baykara M. Endoscopic vesicoureteral reflux correction in transplanted kidneys: does injection technique matter?. J Endourol 2010;24(10):1661-1664.

23. Pichler R, Buttazzoni A, Rehder P, Bartsch G, Steiner $\mathrm{H}$, Oswald J. Endoscopic application of dextranomer/ hyaluronic acid copolymer in the treatment of vesicoureteric reflux after renal transplantation. BJU Int 2011;107(12):1967-1972.

24. Romero NP, Romo MI, Vegas AG, Izquierdo JB, Varela JC, Arteche $\mathrm{AH}$, et al. Deflux injections for vesicoureteral reflux in transplanted kidneys. Transplant Proc 2010;42(8):28922895.

25. Seifert HH, Mazzola B, Ruszat R, Muller A, Steiger J, Bachmann A, et al. Transurethral injection therapy with dextranomer/hyaluronic acid copolymer (Deflux) for treatment of secondary vesicoureteral reflux after renal transplantation. $J$ Endourol 2007;21(11):1357-1360.

26. Cloix P, Gelet A, Desmettre O, Cochat P, Garnier JL, Dubernard JM, et al. Endoscopic treatment of vesicoureteric reflux in transplanted kidneys. Br J Urol 1993;72(1):20-22.

27. Mallet R, Game X, Mouzin M, Sarramon JP, Vaessen C, Malavaud $B$, et al. Symptomatic vesicoureteral reflux in kidney transplantation: results of endoscopic injections of teflon and predictive factors for success. Prog Urol 2003;13(4):598-601.

28. Antonopoulos IM, Piovesan AC, Falci R Jr, Kanashiro $\mathrm{H}_{\text {, }}$ Saito FJ, Nahas WC. Transurethral injection therapy with carbon-coated beads (Durasphere ${ }^{\circledR}$ ) for treatment of recurrent pyelonephritis in kidney transplant patients with vesico-ureteral reflux to the allograft. Clin Transplant 2011;25(2):329-333.

29. Song JC, Hwang HS, Yoon HE, Kim JC, Choi BS, Kim YS, et al. Endoscopic subureteral polydimethylsiloxane injection and prevention of recurrent acute graft pyelonephritis. Nephron Clin Pract 2011;117(4):c385-c3869.

30. Akiki A, Boissier R, Delaporte V, Maurin C, Gaillet S, Karsenty $G$, et al. Endoscopic treatment of symptomatic vesicoureteral reflux after renal transplantation. J Urol 2015;193(1):225-229.

31. Latchamsetty KC, Mital D, Jensik S, Coogan CL. Use of collagen injections for vesicoureteral reflux in transplanted kidneys. Transplant Proc 2003;35(4):1378-1380.

32. Nison L, Hazzan M, Puech P, Bouye S. Endoscopic polydimethylsiloxane injection in prevention of recurrent graft pyelonephritis for transplanted kidney with vesicoureteral reflux. Am J Transplant, 2016;16(Suppl 3). Dirección URL: <http://www.atcmeetingabstracts.com/abstract/ endoscopic-polydimethylsiloxane-injection-in-preventionof-recurrent-graft-pyelonephritis-for-transplanted-kidneywith-vesicoureteral-reflux/.>. [Consulta: 13 de septiembre 2016].

33. Aaronson IA, Rames RA, Greene WB, Walsh LG, Hasal UA, Garen PD. Endoscopic treatment of reflux: migration of Teflon to the lungs and brain. Eur Urol 1993;23(3):394-399. 